\title{
Effects of the Linear Polarization of Polariton Condensates in Their Propagation in Codirectional Couplers
}

\author{
Elena Rozas, Alexey Yulin, Johannes Beierlein, Sebastian Klembt, Sven Höfling, Oleg Egorov, Ulf Peschel,
} I. A. Shelykh, Manuel Gundin, Ignacio Robles-López, M. D. Martín,* and L. Viña

Cite This: ACS Photonics 2021, 8, 2489-2497

Read Online

ABSTRACT: We report on the linear polarization of polariton condensates in a codirectional coupler that allows evanescent coupling between adjacent waveguides. During the condensate's formation, polaritons usually acquire a randomly oriented polarization, however, our results reveal a preferential orientation of the linear polarization along the waveguide propagation path. Furthermore, we observe polarization-dependent intensity oscillations in the output terminal of the coupler, and we identify the mode beating between the linearpolarized eigenmodes as the origin of these oscillations. Our findings provide an insight into the control of the polarization of polariton condensates, paving the way for the development of spin-based polaritonic architectures where condensates propagate over macroscopic distances.

KEYWORDS: polaritons, condensates, microcavities, optical spectroscopy, polarization, directional couplers

$\mathrm{M}$ icrocavity exciton polaritons have been, in the latest years, the subject of numerous investigations given their exceptional properties. ${ }^{1,2}$ These properties, emerging from the strong coupling between their constituents, excitons and photons, allow polaritons to behave as bosonic particles. Their short lifetime, typically of the order of ps, is comparable or even smaller than their respective thermalization times, so in general these particles do not reach thermal equilibrium. However, a condensation similar to a Bose-Einstein one is observed when the particle density is increased. ${ }^{3-7}$ Their very low effective mass $\left(\sim 10^{-4} m_{\mathrm{e}}, m_{\mathrm{e}}\right.$ being the free electron mass) can lead to condensation, even at room temperature. This has indeed been the case in transition metal dichalcogenides, organic semiconductor materials, and lead halide perovskite structures, where the enhancement of the exciton binding energy, characteristic of these compounds, has enabled the observation of strong light-matter coupling, ${ }^{8,9}$ polariton lasing and condensation $^{10-18}$ at room temperature. The ease of use and the flexibility offered by exciton polaritons unwrapped a variety of new proposed devices, such as polariton interferometers, ${ }^{19}$ logic gates, ${ }^{20-22}$ or transistors. ${ }^{23-26}$ Additionally, three-dimensional polariton confinement has been achieved in microcavity pillars. $^{27-31}$ Using two-dimensional lattices of these micropillars, it is possible to emulate graphene and its remarkable properties. $^{32-34}$ All these devices are built using refined lithographic techniques that ensure the polariton confinement along several directions. In the one-dimensional case, only a well-defined longitudinal path along which polariton condensates can travel remains. ${ }^{21,23,35,36}$

In the present work we will focus on semiconductor microcavity couplers. Such optical directional couplers are formed by parallel optical waveguides, closely spaced, so that energy exchange can occur between them. ${ }^{37}$ The coupled power, limited by the mode's overlap in the coupler arms, is determined by the separation between the waveguides, the wavelength, the evanescence of the modes, and the interaction length. These devices have proven to be essential for splitting and combining light in photonic systems and have been used widely in the silicon-on-insulator platform. ${ }^{38}$ Quantum photonic waveguide circuits based on $\mathrm{GaAs} / \mathrm{Ga}_{1-x} \mathrm{Al}_{x} \mathrm{As}$ heterostructures have been demonstrated for the manipulation of quantum states of light. ${ }^{39}$ These devices have also been exploited for guiding surface plasmon polaritons ${ }^{40,41}$ and exciton polaritons. ${ }^{42-44}$ More recently, we have reported on different on-chip routing devices: a counter-directional coupler ${ }^{45}$ and a codirectional coupler for condensates of exciton-polaritons, studying the peculiarities of the polariton propagation ${ }^{46}$ and how this is affected by the waveguides' energetic landscape. ${ }^{47}$ A relevant factor is the spin state of the condensates after polariton's relaxation processes, leading to their condensation. ${ }^{4-52}$ Moreover, a spontaneous buildup of the linear polarization of the emitted light above the polariton condensation threshold has been reported both theoretically $^{53-55}$ and experimentally. ${ }^{4,56-59}$ The orientation of the polarization plane of the emission is pinned to a crystallographic axis of the microcavity. ${ }^{58,59}$ This effect has been effusively observed for trapped polaritons using different trapping mechanisms, such as photonic disorder, ${ }^{60}$ stress, ${ }^{5}$ or

Received: May 20, 2021

Published: August 3, 2021 
annular optical confinement. ${ }^{61-63}$ The study and control of the polarization state of polariton condensates has opened new possibilities of designing and improving spin-based devices. ${ }^{64-70}$ Wire-shaped microcavities are particularly interesting in this respect because, due to their reduced symmetry, each polariton mode shows a polarization splitting into two modes polarized along and perpendicular to the wire axis. ${ }^{71}$

Here, we theoretically and experimentally study the linear polarization of the emission of propagating polariton condensates in polaritonic codirectional couplers. Our results demonstrate a coupling between the adjacent waveguides that is not strongly dependent on polarization. However, we encounter striking polarization-dependent emission oscillations at the output terminal of the coupler. For a given set of perpendicular polarizations we find a phase shift between the oscillation's patterns. To better understand our experimental results, a dissipative Gross-Pitaevskii model is used to describe the polarization dynamics in the device.

\section{EXPERIMENTAL DETAILS}

The sample used in this work is a $\lambda / 2$ cavity with top (bottom)distributed Bragg reflectors consisting of 23 (27) pairs of alternating layers of $\mathrm{Al}_{0.2} \mathrm{Ga}_{0.8} \mathrm{As} / \mathrm{AlAs}$. One stack of four GaAs quantum wells of $7 \mathrm{~nm}$ of nominal width is placed at the antinode of the electromagnetic field inside the cavity, and two additional, identical stacks are embedded in the cavity adjacent mirrors. Low power measurements reveal a $Q$-factor of $\sim 5000$ and a Rabi splitting of $13.9 \mathrm{meV}$. The experiments reported here are performed in a region of the sample with a photon-exciton detuning $\delta \approx-17 \mathrm{meV}$. The sample has been grown by molecular beam epitaxy and processed by reactive ion etching down to the QWs, ${ }^{46}$ creating a pattern of adjacent waveguides where length $(L)$, width $(w)$, and separation $(d)$ have been varied. Figure 1a shows a typical field of couplers, with different

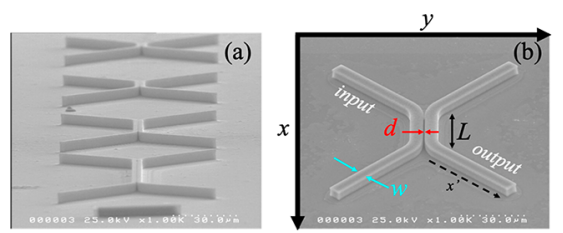

Figure 1. (a) SEM image of a field of directional polariton couplers with different coupling region length $(L)$. (b) SEM image of a directional polariton coupler indicating several parameters: coupling length $(L=$ $20 \mu \mathrm{m})$, waveguide width $(w=6 \mu \mathrm{m})$, and waveguide separation $(d=$ $0.6 \mu \mathrm{m})$. Input and output terminals and the coordinates axis are shown, corresponding to the nomenclature used in the text: $x(y)$ parallel (perpendicular) to the main axis of the waveguides in the coupling region and $x^{\prime}$ along the axes of the input and output terminals at $\mp 45^{\circ}$ with respect to $x$ and $y$, respectively.

coupling lengths, formed by doubly bent waveguides with input and output terminals rotated $\pm 45^{\circ}$ from the longitudinal direction; the geometrical parameters are specified in Figure $1 \mathrm{~b}$. The part of the device where both waveguides remain parallel along the $x$-direction is dubbed coupling region: a few pairs of mirrors left in the region between the two arms enable the evanescent photonic coupling of polaritons between the guides. ${ }^{46}$ For the experiments reported here, the dimensions of the selected device are $L=10 \mu \mathrm{m}, w=2 \mu \mathrm{m}$, and $d=0.2 \mu \mathrm{m}$. The choice of these parameters allows the coupling of a large fraction of polaritons between the arms of the coupler. In our experiments, we nonresonantly pump the input terminal of the coupler with linearly polarized 2 ps pulses from a $\mathrm{Ti}: \mathrm{Al}_{2} \mathrm{O}_{3}$ laser working at $1.664 \mathrm{eV}$, focusing the beam to a $4.5 \mu \mathrm{m}$ diameter spot, with a microscope objective (NA $=0.40, f=4 \mathrm{~mm}$ ), impinging normally to the sample surface. The photoluminescence $(\mathrm{PL})$ is collected through the same objective, while the sample is kept at $12 \mathrm{~K}$ in a coldfinger, He flow cryostat, and detected with a CCD camera attached to a $0.5 \mathrm{~m}$ focal length imaging spectrometer. We ensure that the polariton condensation threshold $\left(12 \mathrm{~kW} / \mathrm{cm}^{2}\right)$ has been exceeded and that condensates propagate along the entire device pumping with a power density of $26 \mathrm{~kW} / \mathrm{cm}^{2}$.

\section{THEORETICAL FRAMEWORK}

To study the dynamics of exciton-polaritons theoretically, we adopt a well-known model describing the coherent polaritons by two complex order parameter functions $\Psi_{r, l}$ for right $(r)$ and left (l) handed circularly polarized polaritons. ${ }^{65,72}$ The coherent polaritons interact with baths of incoherent excitons having different spins. The excitons are characterized by their density $n_{r, l}$. The whole set of equations can be written as

$$
\begin{aligned}
i \hbar \partial_{t} \Psi_{r, l}= & \frac{i \hbar}{2}\left(R n_{r, l}-\gamma_{p}\right) \Psi_{r, l}+\left(V(x, y)+G\left|\Psi_{r, l}\right|^{2}+\tilde{G}\left|\Psi_{l, r}\right|^{2}+G_{R} n_{r, l}\right. \\
& \left.+\widetilde{G_{R}} n_{l, r}\right) \Psi_{r, l}-\frac{\hbar^{2}}{2 m_{\mathrm{eff}}} \nabla^{2} \Psi_{r, l}+\mathrm{B}\left(\partial_{x} \pm i \partial_{y}\right)^{2} \Psi_{l, r}+A_{r, l}(x, y, t) \\
\partial_{t} n_{r, l}= & -\left(\Gamma_{\mathrm{e}}+R\left|\Psi_{r, l}\right|^{2}\right) n_{r, l}+P_{r, l}(x, y, t)
\end{aligned}
$$

In these equations, $R$ is the coupling parameter between the polaritons and the reservoirs of excitons, and $\gamma_{p}$ is the coordinate dependent losses of the coherent polaritons. We assume that the polariton waveguides are formed by microstructuring, creating a coordinate-dependent effective potential, $V$, for the polaritons. It is also considered that the microstructuring affects the transparency of the Bragg mirrors, thus, the losses experienced by polaritons become larger outside the waveguides. $G(\tilde{G})$ and $G_{R}\left(\widetilde{G_{R}}\right)$ denote nonlinear corrections, blue shift, to the effective potential due to interactions between polaritons and between polaritons and incoherent excitons of the same (orthogonal) spin, respectively. $m_{\text {eff }}=\frac{2 m_{\perp} m_{\|}}{m_{\perp}+m_{\|}}$is the reduced effective mass of polaritons, with $m_{\perp}$ and $m_{\|}$the transverse and longitudinal polariton masses, while $\mathrm{B}=\frac{m_{\perp}-m_{\|}}{m_{\perp}+m_{\|}}$defines the strength of TETM splitting (spin-orbit coupling). $\Gamma_{\mathrm{e}}$ accounts for the linear losses in the exciton subsystem, and $P_{r, l}$ is the intensity of the optical pump creating the exciton baths. In the experiments reported here, an incoherent linearly polarized pump has been used in order to create a bath of excitons that are responsible for setting the polaritons in motion. ${ }^{35}$ However, polaritons can also be excited resonantly by coherent light. This kind of excitation provides a simpler scenario in numerical calculations to control the properties of the polaritons; we use it in our numerical simulations to clarify some aspects of polariton dynamics when required. In eqs 1 and 2, this pump is accounted for by the driving force $A_{r, l}(x, y, t)$, the last term on the right-hand side of eq 1.

\section{PROPAGATION OF POLARITONS ALONG THE WAVEGUIDES}

The propagation distance of these condensates following the input waveguide axis $\left(x^{\prime}\right)$ is displayed in Figure 2. The zero position on the horizontal axis corresponds to the excitation 


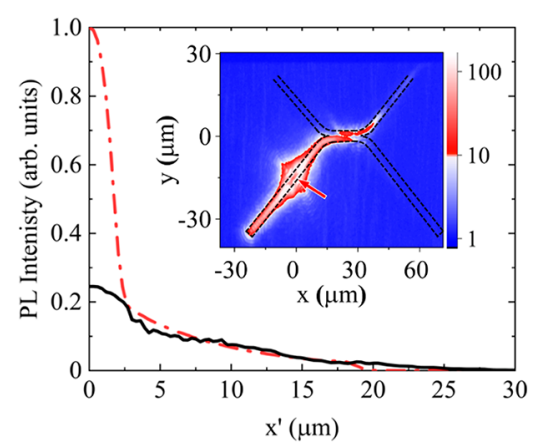

Figure 2. Polariton propagation along the longitudinal axis of the input arm of a polariton coupler. The $x^{\prime}$ origin is placed at the excitation spot. The photoluminescence (PL) intensity (black line) is plotted along the axis of the input terminal, $x^{\prime}$, in the region $(0<x<21,-15<y<0)$ together with the results of the simulation (red line). The inset shows the experimental map of the coupler emission at the condensate energy, $1.583 \mathrm{eV}$, from which the black curve was extracted; the arrow points to the laser excitation spot.

spot. Polariton condensates propagate ballistically away from the excitation spot in the lower input terminal after being generated, as readily seen in the inset, which also shows the coupling to the upper arm. The solid black and dot-dashed red lines depict the experimental PL intensity and the numerical simulation (see below), respectively, for polaritons moving toward the coupling region. Close to the origin, the experimental $\mathrm{PL}$ is much smaller than that of the simulations due to energy filtering performed by the spectrometer to cutoff the bright background of the pumping light having higher frequency and to some limitations of our numerical simulations around the pump area. The parameters for the linear terms in the equations describing polariton dynamics can be extracted from the experimental data, rendering a satisfactory agreement for the linear polariton propagation outside the pump spot, which is the main focus of our paper. However, we cannot get the nonlinear parameters from our experiment and must use rough estimates in their stead. In particular, we assume that the losses are spatially uniform within the waveguides, although at the excitation spot, the losses can be modified by the action of the intense pump beam. Other significant issues for the polariton condensation, as for example phonon-assisted energy relaxation, ${ }^{73}$ are not considered in our simple model. All this accounts for the discrepancy at $x^{\prime}=0$. Away from the excitation area, due to the finite polariton lifetime, the polariton population decays exponentially as it moves away from the excitation area. The simulations describe well the experimental results up to $x^{\prime} \sim 20 \mu \mathrm{m}$, where the intensity droops when reaching the waveguide bend.

Now we describe the condensates propagation in the coupling region and in the output terminal. Since the PL intensity in these regions is substantially lower than in the input terminal, we have spatially filtered the emission, so that the PL from the input terminal is removed. The excitation beam is vertically polarized $\left(\theta_{\mathrm{i}}=90^{\circ}\right)$, that is, parallel to the $y$ axis. We have observed (in contrast with previous works in the literature, which show that the polarization of the nonresonant excitation beam can strongly affect the spinor state of the condensates, ${ }^{51,65}$ and in agreement with numerous reports) that upon condensation the condensate's polarization is independent of the exciting polarization, and it is defined by the crystallographic axis of the microcavity ${ }^{4,58,59}$ and, in our case, by the microstructuring and the presence of the edges of the waveguides. The PL is analyzed using a linear polarizer at different angles, ranging from $\theta_{d}=0^{\circ}$ (i.e., horizontal polarization) up to $180^{\circ}$ in steps of $10^{\circ}$. For simplicity, only a summary of the PL for selected $\theta_{d}$ is shown in Figure 3. Polariton condensates are generated in the bottom-left input terminal; when they arrive to the coupling region, $-5<x<$ $5 \mu \mathrm{m}$, a large fraction of the population is conveyed from the bottom to the top arm. This oscillation of the polariton population between the two arms has been thoroughly discussed in ref 46 , being attributed to the evanescent coupling of two macroscopic wave functions in each arm. It is different to those observed in similar wider structures, where polaritons are traveling, without coupling to the neighboring arm, exhibiting a zigzag trajectory, ${ }^{47}$ that could be interpreted as a manifestation
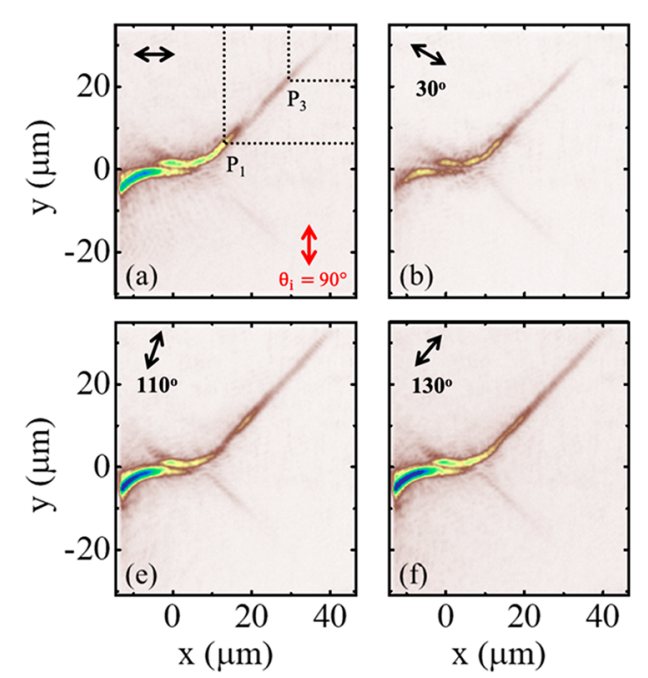
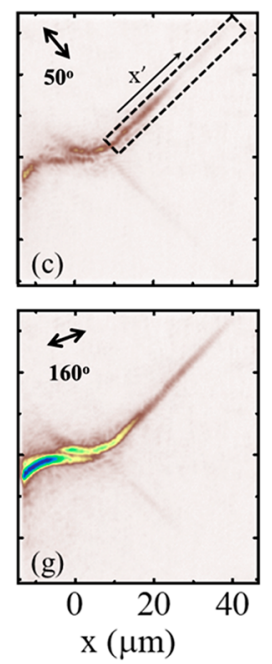

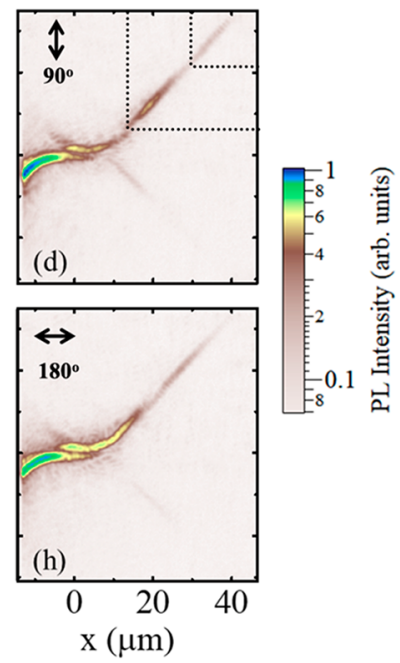

Figure 3. PL of polariton condensates propagating along a coupler. The emission is analyzed at different linear polarizations (black arrows) ranging from $\theta_{d}=0^{\circ}(\mathrm{a})$ to $180^{\circ}(\mathrm{h})$. The excitation at the input terminal (not shown) is performed with a vertically polarized laser beam [denoted by the red arrow in (a)]. The output terminal in which polarization-dependent oscillations are visible for some angles $\theta_{d}$ 's is indicated by a black dashed rectangle in (c); the axis along this terminal is defined as $x^{\prime}$. The positions labeled as $\mathrm{P}_{\mathrm{i}}$ (where $i=1$ and 3 ) mark the maximum amplitude of the aforementioned oscillations for $\theta_{d}=0^{\circ}$. The PL emissions are filtered at an energy of $1.583 \mathrm{eV}$ and depicted in a normalized logarithmic false-color scale. A power density of $26 \mathrm{~kW} / \mathrm{cm}^{2}$ has been used for the measurements. 

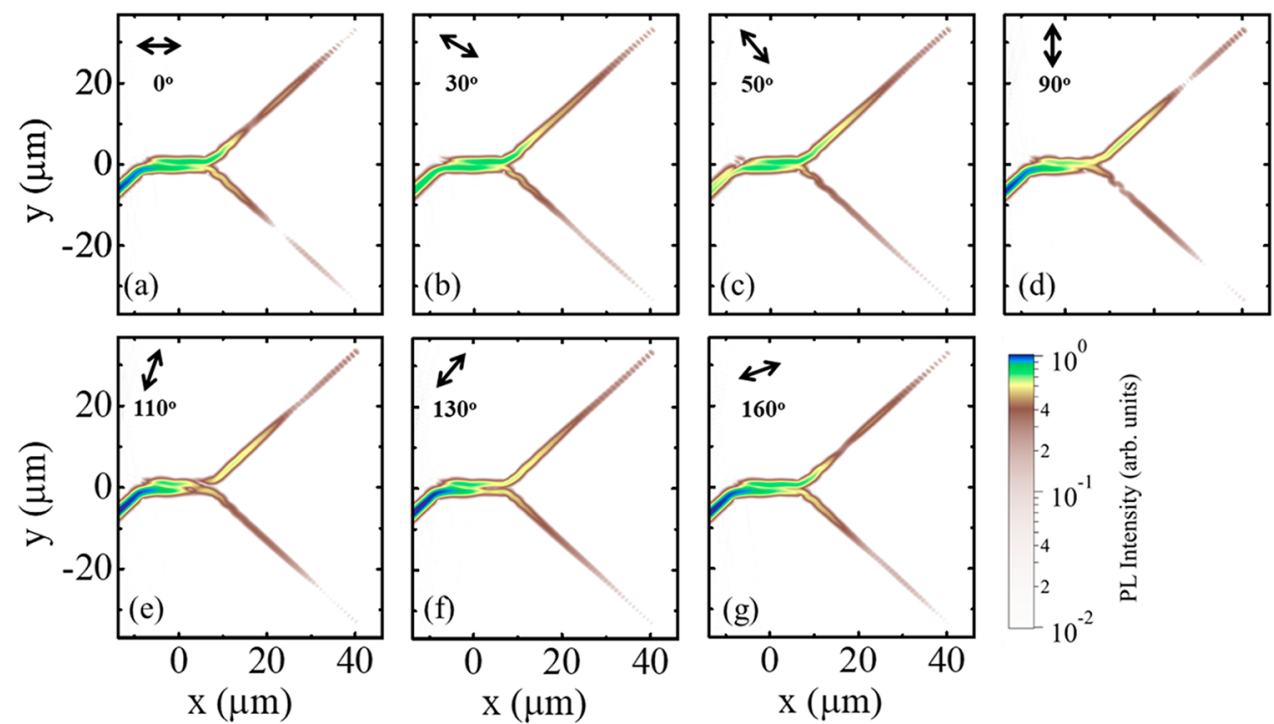

Figure 4. Simulations of the polariton distribution along a coupler. The emission is analyzed at different linear polarizations (black arrows) ranging from $\theta_{d}=0^{\circ}$ (a) to $160^{\circ}(\mathrm{g})$. The excitation is performed with a linearly polarized laser beam at the input terminal (not shown). The density of polaritons is depicted in a normalized logarithmic false-color scale.
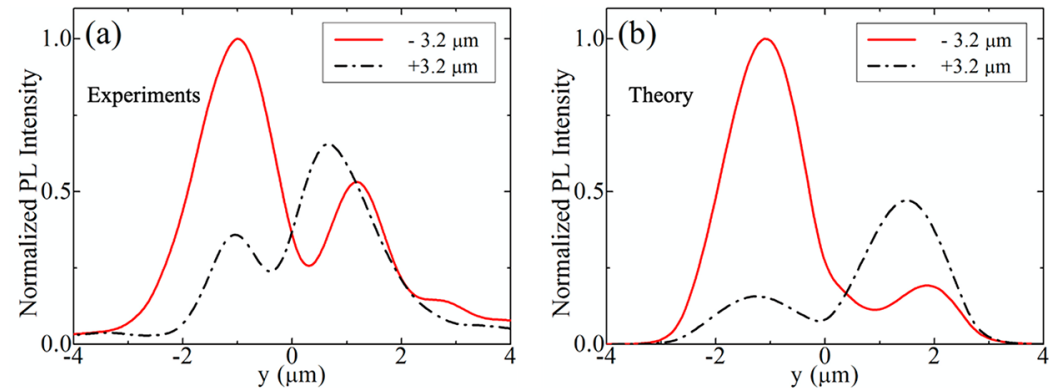

Figure 5. (a) Solid/dash-dotted line depicts the experimental normalized PL intensity vs $y$, the transverse to the axis of the coupling region coordinate, at a position close to the entrance/exit of the coupling region, $-3.2 \mu \mathrm{m} /+3.2 \mu \mathrm{m}$, for $\theta_{d}=0^{\circ}$. (b) Corresponding simulated polariton densities at the same positions of the coupler as those shown in panel (a).

of polaritons experiencing zitterbewegung. ${ }^{74}$ After the coupling, polaritons continue propagating throughout the top arm until the edge of the waveguide at the output terminal, while only a minor fraction of the population remains in the bottom arm. By increasing either the length $(L)$ or the spacing $(d)$ between the arms, the fraction of coupled polaritons can be controlled. ${ }^{46}$ Drastic intensity variations along the device are observed when the polarization of the emission is analyzed. We find a considerably large intensity when the polarization is analyzed at $0^{\circ}$. By contrast, a remarkable intensity reduction is observed around and above $30^{\circ}$. A further increase of $\theta_{d}$ results in a slow PL recovery for $\theta_{d} \gtrsim 90^{\circ}$.

Furthermore, a conspicuous additional effect is observed at the output terminal. The PL on this terminal displays two local maxima at $P_{1}=(13,7)$ and $P_{3}=(30,22) \mu \mathrm{m}$ when the polarization is filtered at $0^{\circ}$. This is in stark contrast with the emission observed at $\theta_{d}=90^{\circ}$, in which local minima appear at the same set of coordinates. These intensity oscillations in the $\mathrm{PL}$ do not exist for intermediate polarizations (see for instance $50^{\circ}$ and $130^{\circ}$ ): the emission shows just an exponential decay with propagation distance along the output terminal. This behavior is independent of the linear polarization of the excitation laser as borne out by our experiments, since the nonresonant excitation conditions in our case guarantee the erasing of polariton's spin memory during the relaxation processes (see also Figure S2).

Let us now discuss how the theoretical model introduced above describes the effects observed in the experiment. We performed numerical simulations of the waveguides with the experimental geometrical parameters and with a depth and width of the effective polariton potential providing the width of the fundamental mode (full width at half-maximum of the intensity) to be very close to $1 \mu \mathrm{m}$ : the width of the mode in the modeled waveguide is equal to the width of the fundamental mode of an infinitely deep rectangular potential of $2 \mu \mathrm{m}$ wide. In the numerical simulations, we use a random noise of low intensity as initial conditions and excite the system by an incoherent pump creating a bath of excitons, which eventually create the polariton condensates. The results of the numerical simulations shown in Figure 4 attest to a good qualitative agreement with the experiments: they reproduce the observation that the polariton condensate is preferentially polarized along the direction of the waveguide.

Let us mention that, for our experimental conditions, the polaritons propagation is linear away from the excitation spot. The polarization dynamics observed in the experiment and in the numerical simulations is directly related to the optical spin Hall effect appearing because of the TE-TM splitting. ${ }^{65,75}$ Glazov et al. pioneering work ${ }^{75}$ reports a temporal precession of 
the pseudospin of the polaritons excited by an optical pulse. This precession takes place because of the TE-TM splitting in the dispersion relation. This splitting also affects the spatial evolution of the polaritons polarization when they propagate under cw-excitation conditions. The confinement of the polaritons inside the microstructured waveguide affects both the eigenmodes and the dispersion relations. For our narrow structures only the effects in the fundamental mode need to be considered. The details of the theory necessary to understand the linear polariton propagation are given in the Supporting Information (SI).

Additionally, the numerical modeling replicates qualitatively the experiments bearing out the tunneling of polaritons from the lower to the upper waveguide in the coupling region. Figure 5 presents a comparison between the experimental results [panel (a)] and the calculations [panel (b)]: the normalized PL intensity is plotted as a function of $y$, the transverse to the axis of the coupling region coordinate, for a position close to the entrance/exit $(-3.2 /+3.2 \mu \mathrm{m})$ of that region with a solid/dashdotted line (for $\theta_{d}=0$ ). Note that $y=0$ marks the center of the gap between the arms, therefore, the signal from $y<0$ and $y>0$ arises from the pumped and coupled arm, respectively. It is apparent that, at the entrance, a larger polariton population is present in the pumped arm of the coupler $(y<0)$ than that in the coupled arm $(y>0)$, both in the experiments and the simulations. This situation is reversed toward the exit, where the population becomes larger in the coupled arm.

The amount of polaritons transferred to the upper arm depends on the height and the width of the potential separating the upper and the lower arms as well as on the dissipation rate of the propagating polaritons, which experimentally is greatly influenced by sample inhomogeneities. The lack of a full quantitative agreement between the theoretical and the experimental polariton transfer can be attributed to the not perfect fitting of the experimental polariton dissipation rate and the height of the effective confinement potential used in the simulations.

It should also be stated that, both in the experiments and theory, the population transfer is not strongly polarization dependent and $65( \pm 4) \%$ of the polaritons are transferred in the coupling region, regardless of their polarization, for our specific sample and experimental conditions. The coupling region where the waveguides go parallel to each other is short $(10 \mu \mathrm{m})$ and the distance between the waveguides is much shorter; therefore, the tunneling length is less than $10 \mu \mathrm{m}$ and most of the polaritons are transferred from the lower to the upper waveguide. Furthermore, the interaction between the different polarizations is relatively weak, so considerably longer propagation distances would be required to observe polarization effects in the tunneling of polaritons. From a theoretical point of view, the effect of the coupling between the polarizations and the waveguides produces four eigenmodes having different propagation constants for the same frequency. One can anticipate that this should result in a complex behavior of the polaritons bouncing between the waveguide and changing the polarization state. However, for our structures and experimental conditions, the effects originating from the coupling between the waveguides are much stronger than those caused by the spin-orbit interaction. Thus, the consequences of the latter on the propagation within the relatively short coupling area can be neglected.

Finally, and most importantly for the present work, the polarization beating seen in the output terminal of the upper waveguide is unmistakably obtained in the simulations. This effect originates from the TE-TM splitting of the polaritons: it is theoretically discussed in detail in the next section and in the SI.

\section{POLARITON'S POLARIZATION DYNAMICS}

To quantitatively describe the polarized PL, we focus now on two regions of the coupler: the coupling region and the output terminal. In the coupling region, the PL intensity is found experimentally to be maximum for $\theta_{d}=0^{\circ}\left(=180^{\circ}\right)$, that is, when the polarization is horizontal, parallel to the longitudinal axis in this region. On the contrary, the intensity drops with increasing $\theta_{d}$ reaching its minimum at $\sim 45^{\circ}$. A further increase of $\theta_{d}$ leads to a partial recovery of the emission, resulting in a $\mathrm{PL}$ for $\theta_{d}=90^{\circ}$ (vertical polarization), $\sim 50 \%$ lower than that of $\theta_{d}=0^{\circ}$. These results reveal a preferential orientation of the polarization along the longitudinal axis of the waveguide. For larger $\theta_{d}$, being the polarized emission direction closer to the orientation of the pumped (input) terminal, the PL intensity in the coupled region becomes high again as a consequence of a larger population with this polarization in the input terminal. This is readily seen in the polarization maps compiled in Figure 6. Panel (a)/(c) shows the
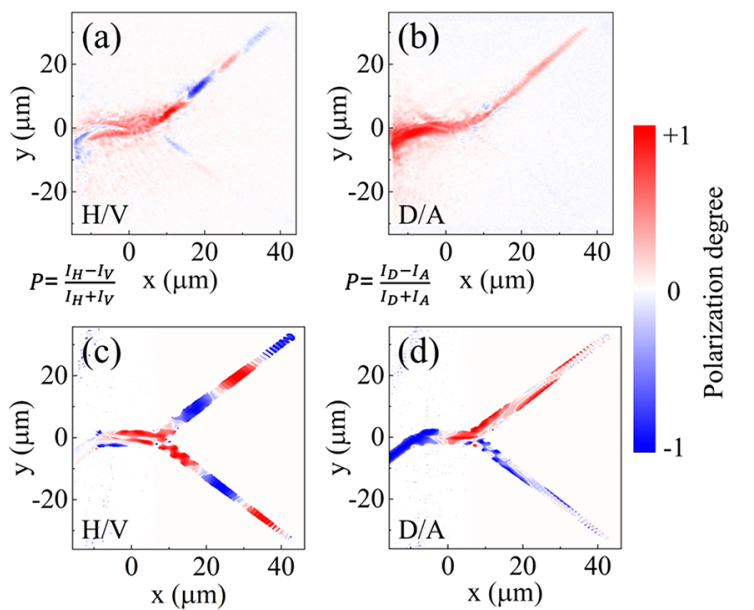

Figure 6. (a) Spatial maps of the polariton polarization degree, $P=$ $\frac{I_{\mathrm{H}}-I_{\mathrm{V}}}{I_{\mathrm{H}}+I_{\mathrm{V}}}$, for $\theta_{d}=0^{\circ}(\mathrm{H})$ and $\theta_{d}=90^{\circ}(\mathrm{V})$ polarizations of the emission in the coupler. (b) Corresponding maps for $\theta_{d}=50^{\circ}(\mathrm{D})$ and $\theta_{d}=130^{\circ}$ (A) rendering $P=\frac{I_{\mathrm{D}}-I_{\mathrm{A}}}{I_{\mathrm{D}}+I_{\mathrm{A}}}$. (c, d) Analogous degrees of polarization obtained from the simulated polariton densities for the same analyzer angles as those presented in (a) and (b), respectively. The degree of polarization is plotted in a false color scale with red (blue) corresponding to positive (negative) values.

experimental/simulated degree of polarization, defined as $P=$ $\frac{I_{\mathrm{H}}-I_{\mathrm{V}}}{I_{\mathrm{H}}+I_{\mathrm{V}}}$, where $I_{\mathrm{H}}\left(I_{\mathrm{V}}\right)$ is the PL intensity for $\theta_{d}=0^{\circ}\left(90^{\circ}\right)$, and demonstrates the preferential polarization orientation in the coupling region, where a positive degree of polarization (red coded values) is obtained both in the experiments and the simulations. A positive degree of polarization in this region is also obtained in the diagonal basis, as depicted in panel (b)/(d) for $P=\frac{I_{\mathrm{D}}-I_{\mathrm{A}}}{I_{\mathrm{D}}+I_{\mathrm{A}}}$ obtained in the experiments/simulations; in this case, $I_{\mathrm{D}}\left(I_{\mathrm{A}}\right)$ is the PL intensity for $\theta_{d}=50^{\circ}\left(130^{\circ}\right)$.

We focus now on the polarization-dependent oscillations at the output terminal. These oscillations are clearly seen as a sign change of $P$ (going alternatively to red-and blue-coded values), 
both in the experiments and in the simulations, in panels (a) and (c) of Figure 6, that show the polarization degree in the H/V basis. However, they vanish when the polarization is analyzed in the D/A polarization basis [see panels (b) and (d)]. For a better understanding of this effect, PL profiles have been extracted along the $x^{\prime}$ direction of the terminal, in the region marked with a dashed line in Figure 3c. Figure 7 summarizes the experimental

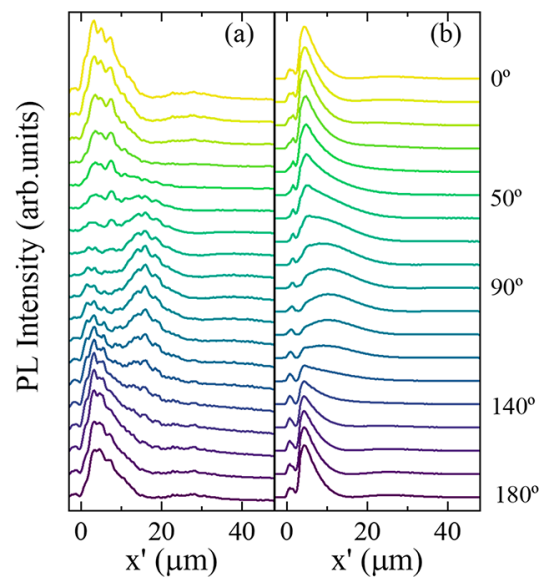

Figure 7. (a) PL intensity, integrated along the transverse coordinate in the output terminal of the coupler, vs distance, measured along the longitudinal axis at this terminal, for different analyzer angles, ranging from $\theta_{d}=0^{\circ}$ (top) to $180^{\circ}$ (bottom) in steps of $10^{\circ}$. (b) Corresponding simulated polariton densities for the same $\theta_{d}$ 's.

(a) and simulated (b) emission profiles for different polarizations between $\theta_{d}=0^{\circ}$ and $180^{\circ}$ in steps of $10^{\circ}$. The zero position denotes the beginning of the output terminal, while the end of the structure is located at $x^{\prime} \sim 45 \mu \mathrm{m}$. A transition between different patterns can be distinguished. For $\theta_{d}$ close to $0^{\circ}$ and $180^{\circ}$, beatings separated by $\sim 24 \mu \mathrm{m}$ are observed, with the maxima at $x_{1}{ }^{\prime} \sim 4 \mu \mathrm{m}$ and $x_{3}{ }^{\prime} \sim 28 \mu \mathrm{m}$. In contrast, for $\theta_{d}$ close to $90^{\circ}$, a weak signal is obtained at $x_{1}{ }^{\prime}$ being the maximum now at $x_{2}{ }^{\prime} \sim 16 \mu \mathrm{m}$, that is, out of phase from $x_{1}{ }^{\prime}$ by half the beating distance $(12 \mu \mathrm{m})$.

We can explain these polarization beatings by considering the TE-TM splitting in the waveguide and therefore taking into account the different group velocities for each polarization. In the framework of the mathematical model introduced above, the splitting lifts the degeneracy between the eigenmode polarized along (TE) and that polarized perpendicularly (TM) to the waveguide axis. ${ }^{76}$ At a given frequency, these two modes have different wavevectors, and therefore, if both modes are excited, interference fringes are obtained when the polarization of the propagating polaritons is a linear combination of those of the eigenmodes. The beating period is given by $L_{\text {beating }}=\frac{2 \pi}{\left|k_{\|}-k_{\perp}\right|}$, where $k_{\|}$and $k_{\perp}$ are the wavevectors of the TE and TM modes, respectively. This is the case along the output terminal for $\theta_{d}=$ $0^{\circ}$ and $90^{\circ}$, which are linear combinations of the $\mathrm{TM}\left(45^{\circ}\right)$ and TE $\left(135^{\circ}\right)$ modes in this part of the coupler, where clear beatings are observed both in the experiments and simulations. However, the beats are absent for $\theta_{d} \sim 45^{\circ}$ and $\sim 135^{\circ}$.

We would like to mention here that PL intensity fringes having a short spatial period are also clearly seen at the ends of the waveguides. These short-period oscillations are visible since polariton coherence is preserved during their propagation. ${ }^{77,78}$ They originate from the reflection of the polaritons at the waveguide end, resulting in the formation of counter- propagating polariton waves that interfere with the incoming ones. ${ }^{4}$

Finally, let us stress that the theory presented here does not aim to give a comprehensive description of the quite large variety of polarization effects that can be observed in exciton polariton systems, but to back our experimental results. A systematic analysis of different linear and nonlinear regimes of polarization beats is a challenge of great interest, but is out of the scope of the present paper.

\section{CONCLUSIONS}

In summary, we have evidenced the rich phenomenology of polariton propagation in codirectional couplers when the population is analyzed into its linearly polarized components. A detailed analysis of the PL has been accomplished by mapping the polarized-dependent emission at the condensate's energy. Two different sections of the coupler have been studied in detail, the coupling region and the output terminal. The former region shows a transfer of polariton population from the pumped- to the coupled-arm of the device that is not strongly polarization dependent. In the latter region, on the contrary, polarizationdependent oscillations emerging from the TE-TM splitting of the fundamental modes of the waveguide have been found. In these codirectional couplers, based on waveguide microcavities, which provide a polarization-dependent mode spectrum, the polariton spin gains crucial importance. Our work paves the way for the use of polariton codirectional couplers, taking advantage of the changes of the polariton's polarization state during their propagation. Further effects of the geometry of the couplers on the spin of the polariton condensates, such as the influence of the bends on the circular polarization, are currently under investigation.

\section{ASSOCIATED CONTENT}

\section{Supporting Information}

The Supporting Information is available free of charge at https://pubs.acs.org/doi/10.1021/acsphotonics.1c00746.

SEM image of the microcavity structure together with refractive index and electromagnetic field profiles. Additional experiments on polarization beatings at the output terminal. Simulations of the polariton dynamics. Excitation by either a coherent or incoherent pump (PDF) Movie of polarization degree of propagating polaritons (MP4)

\section{AUTHOR INFORMATION}

\section{Corresponding Author}

M. D. Martín - Departamento de Física de Materiales and Instituto Nicolás Cabrera, Universidad Autónoma de Madrid, 28049 Madrid, Spain; 이이이.org/0000-0003-2256-1418; Email: dolores.martin@uam.es

\section{Authors}

Elena Rozas - Departamento de Física de Materiales and Instituto Nicolás Cabrera, Universidad Autónoma de Madrid, 28049 Madrid, Spain

Alexey Yulin - Faculty of Physics and Engineering, ITMO University, St. Petersburg 197101, Russia

Johannes Beierlein - Technische Physik, Wilhelm-ConradRöntgen-Research Center for Complex Material Systems, and Würzburg-Dresden Cluster of Excellence ct.qmat, Universität Würzburg, D-97074 Würzburg, Germany 
Sebastian Klembt - Technische Physik, Wilhelm-ConradRöntgen-Research Center for Complex Material Systems, and Würzburg-Dresden Cluster of Excellence ct.qmat, Universität Würzburg, D-97074 Würzburg, Germany

Sven Höfling - Technische Physik, Wilhelm-Conrad-RöntgenResearch Center for Complex Material Systems, and Würzburg-Dresden Cluster of Excellence ct.qmat, Universität Würzburg, D-97074 Würzburg, Germany; SUPA, School of Physics and Astronomy, University of St. Andrews, St. Andrews KY16 9SS, United Kingdom

Oleg Egorov - Institute of Condensed Matter Theory and Optics, Friedrich-Schiller-University Jena, D-07743 Jena, Germany

Ulf Peschel - Institute of Condensed Matter Theory and Optics, Friedrich-Schiller-University Jena, D-07743 Jena, Germany

I. A. Shelykh - Faculty of Physics and Engineering, ITMO University, St. Petersburg 197101, Russia; Science Institute, University of Iceland, Reykjavik IS-107, Iceland

Manuel Gundin - Departamento de Física de Materiales, Universidad Autónoma de Madrid, 28049 Madrid, Spain

Ignacio Robles-López - Departamento de Física de Materiales and Instituto Nicolás Cabrera, Universidad Autónoma de Madrid, 28049 Madrid, Spain

L. Viña - Departamento de Física de Materiales, Instituto Nicolás Cabrera, and Instituto de Física de la Materia Condensada, Universidad Autónoma de Madrid, 28049 Madrid, Spain; (1) orcid.org/0000-0002-6376-6703

Complete contact information is available at: https://pubs.acs.org/10.1021/acsphotonics.1c00746

\section{Funding}

This work has been partly supported by the Spanish MINECO Grant Nos. MAT2017-83722-R and PID2020-113445GB-I00. A.Y. and I.A.S. were financially supported by the Ministry of Science and Higher Education of the Russian Federation through Megagrant Number 14.Y26.31.0015 and Goszadanie No. 2019-1246. I.A.S. acknowledges also the support from the Icelandic research fund, Grant No. 163082-051. The Würzburg and Jena group acknowledges financial support within the DFG Project Nos. PE 523/18-1 and KL3124/2-1. The Würzburg group acknowledges financial support by the German Research Foundation (DFG) under Germany's Excellence StrategyEXC2147 "ct.qmat" (Project No. 390858490) and is grateful for support by the state of Bavaria.

Notes

The authors declare no competing financial interest.

\section{ACKNOWLEDGMENTS}

We thank C. Schneider and H. Suchomel for sample growth and useful discussions.

\section{REFERENCES}

(1) Weisbuch, C.; Nishioka, M.; Ishikawa, A.; Arakawa, Y. Observation of the coupled exciton-photon mode splitting in a semiconductor quantum microcavity. Phys. Rev. Lett. 1992, 69, 3314-3317.

(2) Kavokin, A.; Baumberg, J.; Malpuech, G.; Laussy, F. Microcavities, 2nd ed.; Oxford Science Publications: Oxford, 2017.

(3) Dang, L. S.; Heger, D.; André, R.; Bœuf, F.; Romestain, R. Stimulation of polariton photoluminescence in semiconductor microcavity. Phys. Rev. Lett. 1998, 81, 3920-3923.

(4) Kasprzak, J.; Richard, M.; Kundermann, S.; Baas, A.; Jeambrun, P.; Keeling, J.; Marchetti, F. M.; Szymańska, M. H.; André, R.; Staehli, J. L.;
Savona, V.; Littlewood, P. B.; Deveaud, B.; Dang, L. S. Bose-Einstein condensation of exciton polaritons. Nature 2006, 443, 409-414.

(5) Balili, R.; Hartwell, V.; Snoke, D.; Pfeiffer, L.; West, K. BoseEinstein condensation of microcavity polaritons in a trap. Science 2007, 316, 1007-1010.

(6) Deng, H.; Haug, H.; Yamamoto, Y. Exciton-polariton BoseEinstein condensation. Rev. Mod. Phys. 2010, 82, 1489-1537.

(7) Byrnes, T.; Kim, N.; Yamamoto, Y. Exciton-polariton condensates. Nat. Phys. 2014, 10, 803-813.

(8) Liu, X.; Galfsky, T.; Sun, Z.; Xia, F.; Lin, E.; Lee, Y.; Kéna-Cohen, S.; Menon, V. M. Strong light-matter coupling in two-dimensional atomic crystals. Nat. Photonics 2015, 9, 30-34.

(9) Ardizzone, V.; de Marco, L.; de Giorgi, M.; Dominici, L.; Ballarini, D.; Sanvitto, D. Emerging 2D materials for room-temperature polaritonics. Nanophotonics 2019, 8, 1547-1558.

(10) Kéna-Cohen, S.; Forrest, S. R. Room-temperature polariton lasing in an organic single-crystal microcavity. Nat. Photonics 2010, 4, $371-375$.

(11) Daskalakis, K. S.; Maier, S. A.; Murray, R.; Kéna-Cohen, S. Nonlinear interactions in an organic polariton condensate. Nat. Mater. 2014, 13, 271-278.

(12) Plumhof, J. D.; Stöferle, T.; Mai, L.; Scherf, U.; Mahrt, R. F. Room-temperature Bose-Einstein condensation of cavity excitonpolaritons in a polymer. Nat. Mater. 2014, 13, 247-252.

(13) Su, R.; Diederichs, C.; Wang, J.; Liew, T. C. H.; Zhao, J.; Liu, S.; $\mathrm{Xu}, \mathrm{W}$.; Chen, Z.; Xiong, Q. Room-temperature polariton lasing in allinorganic perovskite nanoplatelets. Nano Lett. 2017, 17, 3982-3988.

(14) Su, R.; Wang, J.; Zhao, J.; Xing, J.; Zhao, W.; Diederichs, C.; Liew, T. C. H.; Xiong, Q. Room temperature long-range coherent exciton polariton condensate flow in lead halide perovskites. Sci. Adv. 2018, 4, No. eaau0244.

(15) Dusel, M.; Betzold, S.; Egorov, O. A.; Klembt, S.; Ohmer, J.; Fischer, U.; Höfling, S.; Schneider, C. Room temperature organic exciton-polariton condensate in a lattice. Nat. Commun. 2020, 11, 2863.

(16) Su, R.; Ghosh, S.; Wang, J.; Liu, S.; Diederichs, C.; Liew, T. C. H.; Xiong, Q. Observation of exciton polariton condensation in a perovskite lattice at room temperature. Nat. Phys. 2020, 16, 301-306.

(17) Anton-Solanas, C.; Waldherr, M.; Klaas, M.; Suchomel, H.; Harder, T. H.; Cai, H.; Sedov, E.; Klembt, S.; Kavokin, A. V.; Tongay, S.; Watanabe, K.; Taniguchi, T.; Höfling, S.; Schneider, C. Bosonic condensation of exciton-polaritons in an atomically thin crystal. Nat. Mater. 2021, na DOI: 10.1038/s41563-021-01000-8.

(18) Shan, H.; Lackner, L.; Han, B.; Sedov, E.; Rupprecht, C.; Knopf, H.; Eilenberger, F.; Yumigeta, K.; Watanabe, K.; Taniguchi, T.; Klembt, S.; Höfling, S.; Kavokin, A. V.; Tongay, S.; Schneider, C.; AntónSolanas, C. Coherent light emission of exciton-polaritons in an atomically thin crystal at room temperature. arXiv:2103.10459 [condmat.mes-hall] 2021, No. na.

(19) Sturm, C.; Tanese, D.; Nguyen, H. S.; Flayac, H.; Galopin, E.; Lemaître, A.; Sagnes, I.; Solnyshkov, D.; Amo, A.; Malpuech, G.; Bloch, J. All-optical phase modulation in a cavity-polariton Mach-Zehnder interferometer. Nat. Commun. 2014, 5, 3278.

(20) Espinosa-Ortega, T.; Liew, T. C. H. A complete architecture of integrated photonic circuits based on AND and NOT logic gates of exciton-polaritons in semiconductor microcavities. Phys. Rev. B: Condens. Matter Mater. Phys. 2013, 87, 195305.

(21) Antón, C.; Liew, T. C. H.; Cuadra, J.; Martín, M. D.; Eldridge, P. S.; Hatzopoulos, Z.; Stavrinidis, G.; Savvidis, P. G.; Viña, L. Quantum reflections and shunting of polariton condensate wave trains: Implementation of a logic AND gate. Phys. Rev. B: Condens. Matter Mater. Phys. 2013, 88, 245307.

(22) Zasedatelev, A.; Baranikov, A. V.; Sannikov, D.; Urbonas, D.; Scarimuto, F.; Shishkov, V. Yu.; Andrianov, E. S.; Lozovik, Y. E.; Scherf, U.; Stöferle, T.; Mahrt, R. F.; Lagoudakis, P. G. Organic single-photon switch. arXiv:2005.05811v2 [cond-mat.mes-hall] 2021, No. na.

(23) Gao, T.; Eldridge, P. S.; Liew, T. C. H.; Tsintzos, S. I.; Stavrinidis, G.; Deligeorgis, G.; Hatzopoulos, Z.; Savvidis, P. G. Polariton condensate transistor switch. Phys. Rev. B: Condens. Matter Mater. Phys. 2012, 85, 235102. 
(24) Antón, C.; Liew, T. C. H.; Tosi, G.; Martín, M. D.; Gao, T.; Hatzopoulos, Z.; Eldridge, P. S.; Savvidis, P. G.; Viña, L. Dynamics of a polariton condensate transistor switch. Appl. Phys. Lett. 2012, 101, 261116.

(25) Ballarini, D.; de Giorgi, M.; Cancellieri, E.; Houdré, R.; Giacobino, E.; Cingolani, R.; Bramati, A.; Gigli, G.; Sanvitto, D. Alloptical polariton transistor. Nat. Commun. 2013, 4, 1778.

(26) Zasedatelev, A. V.; Baranikov, A. V.; Urbonas, D.; Scafirimuto, F.; Scherf, U.; Stöferle, T.; Mahrt, R. F.; Lagoudakis, P. G. A roomtemperature organic polariton transistor. Nat. Photonics 2019, 13, 378383.

(27) Bajoni, D.; Peter, E.; Senellart, P.; Smirr, J. L.; Sagnes, I.; Lemaitre, A.; Bloch, J. Polariton parametric luminescence in a single micropillar. Appl. Phys. Lett. 2007, 90, 051107.

(28) Galbiati, M.; Ferrier, L.; Solnyshkov, D. D.; Tanese, D.; Wertz, E.; Amo, A.; Abbarchi, M.; Senellart, P.; Sagnes, I.; Lemaître, A.; Galopin, E.; Malpuech, G.; Bloch, J. Polariton condensation in photonic molecules. Phys. Rev. Lett. 2012, 108, 126403.

(29) Antón, C.; Solnyshkov, D.; Tosi, G.; Martín, M. D.; Hatzopoulos, Z.; Deligeorgis, G.; Savvidis, P. G.; Malpuech, G.; Viña, L. Ignition and formation dynamics of a polariton condensate on a semiconductor microcavity pillar. Phys. Rev. B: Condens. Matter Mater. Phys. 2014, 90, 155311.

(30) Schneider, C.; Gold, P.; Reitzenstein, S.; Höfling, S.; Kamp, M. Quantum dot micropillar cavities with quality factors exceeding 250000. Appl. Phys. B: Lasers Opt. 2016, 122, 19.

(31) Bajoni, D.; Senellart, P.; Wertz, E.; Sagnes, I.; Miard, A.; Lemaitre, A.; Bloch, J. Polariton laser using single micropillarGaAsGaAlAs semiconductor cavities. Phys. Rev. Lett. 2008, 100, 047401.

(32) Jacqmin, T.; Carusotto, I.; Sagnes, I.; Abbarchi, M.; Solnyshkov, D.; Malpuech, G.; Galopin, E.; Lemaître, A.; Bloch, J.; Amo, A. Direct observation of Dirac cones and a flatband in a honeycomb lattice for polaritons. Phys. Rev. Lett. 2014, 112, 116402.

(33) Jamadi, O.; Rozas, E.; Salerno, G.; Milicevic, M.; Ozawa, T.; Sagnes, I.; Lemaitre, A.; Le Gratiet, L.; Harouri, A.; Carusotto, I.; Bloch, J.; Amo, A. Direct observation of photonic Landau levels and helical edge states in strained honeycomb lattices. Light: Sci. Appl. 2020, 9, 144.

(34) Klembt, S.; Harder, T. H.; Egorov, O. A.; Winkler, K.; Ge, R.; Bandres, M. A.; Emmerling, M.; Worschech, L.; Liew, T. C. H.; Segev, M.; Schneider, C.; Höfling, S. Exciton-polariton topological insulator. Nature 2018, 562, 552-556.

(35) Wertz, E.; Ferrier, L.; Solnyshkov, D. D.; Johne, R.; Sanvitto, D.; Lemaître, A.; Sagnes, I.; Grousson, R.; Kavokin, A. V.; Senellart, P.; Malpuech, G.; Bloch, J. Spontaneous formation and optical manipulation of extended polariton condensates. Nat. Phys. 2010, 6, 860-864.

(36) Wertz, E.; Amo, A.; Solnyshkov, D. D.; Ferrier, L.; Liew, T. C. H.; Sanvitto, D.; Senellart, P.; Sagnes, I.; Lemaitre, A.; Kavokin, A. V.; Malpuech, G.; Bloch, J. Propagation and Amplification Dynamics of 1D Polariton Condensates. Phys. Rev. Lett. 2012, 109, 216404.

(37) Ebeling, K. J. Directional Couplers. Integrated Optoelectronics; Springer Verlag: Berlin, 1993; Chapter 6.

(38) Lu, Z.; Yun, H.; Wang, Y.; Chen, Z.; Zhang, F.; Jaeger, N. A. F.; Chrostowski, L. Broadband silicon photonic directional coupler using asymmetric-waveguide based phase control. Opt. Express 2015, 23, 3795-3808 and references therein.

(39) Wang, J.; Santamato, A.; Jiang, P.; Bonneau, D.; Engin, E.; Silverstone, J. W.; Lermer, M.; Beetz, J.; Kamp, M.; Höfling, S.; Tanner, M. G.; Natarajan, C. M.; Hadfield, R. H.; Dorenbos, S. N.; Zwiller, V.; O'Brien, J. L.; Thompson, M. G. Gallium arsenide (GaAs) quantum photonic waveguide circuits. Opt. Commun. 2014, 327, 49-55.

(40) Zhang, Y.; Xu, Y.; Tian, C.; Xu, Q.; Zhang, X.; Li, Y.; Zhang, X.; Han, J.; Zhang, W. Terahertz spoof surface-plasmon-polariton subwavelength waveguide. Photonics Res. 2018, 6, 18-23.

(41) Pan, M.-Y.; Lin, E.-H.; Wang, L.; Wei, P.-K. Spectral and mode properties of surface plasmon polariton waveguides studied by nearfield excitation and leakage-mode radiation measurement. Nanoscale Res. Lett. 2014, 9, 430.
(42) Flayac, H.; Savenko, I. G. An exciton-polariton mediated alloptical router. Appl. Phys. Lett. 2013, 103, 201105.

(43) Marsault, F.; Nguyen, H. S.; Tanese, D.; Lemaître, A.; Galopin, E.; Sagnes, I.; Amo, A.; Bloch, J. Operation of a semiconductor microcavity under electric excitation. Appl. Phys. Lett. 2015, 107, 201115.

(44) Liao, L.; Ling, Y.; Luo, S.; Zhang, Z.; Wang, J.; Chen, Z. Propagation of a polariton condensate in a one-dimensional microwire at room temperature. Appl. Phys. Express 2019, 12, 052009.

(45) Klaas, M.; Beierlein, J.; Rozas, E.; Klembt, S.; Suchomel, H.; Harder, T. H.; Winkler, K.; Emmerling, M.; Flayac, H.; Martín, M. D.; Viña, L.; Höfling, S.; Schneider, C. Counter-directional polariton coupler. Appl. Phys. Lett. 2019, 114, 061102.

(46) Beierlein, J.; Rozas, E.; Egorov, O. A.; Klaas, M.; Yulin, A.; Suchomel, H.; Harder, T. H.; Emmerling, M.; Martin, M. D.; Shelykh, I. A.; Schneider, C.; Peschel, U.; Vina, L.; Hofling, S.; Klembt, S. Propagative oscillations in codirectional polariton waveguide couplers. Phys. Rev. Lett. 2021, 126, 075302.

(47) Rozas, E.; Beierlein, J.; Yulin, A.; Klaas, M.; Suchomel, H.; Egorov, O.; Shelykh, I. A.; Peschel, U.; Schneider, C.; Klembt, S.; Höfling, S.; Martín, M. D.; Viña, L. Impact of the energetic landscape on polariton condensates' propagation along a coupler. Adv. Opt. Mater. 2020, 8, 2000650.

(48) Martín, M. D.; Aichmayr, G.; Viña, L.; André, R. Polarization control of the nonlinear emission of semiconductor microcavities. Phys. Rev. Lett. 2002, 89, 077402.

(49) Lagoudakis, P. G.; Savvidis, P. G.; Baumberg, J. J.; Whittaker, D. M.; Eastham, P. R.; Skolnick, M. S.; Roberts, J. S. Stimulated spin dynamics of polaritons in semiconductor microcavities. Phys. Rev. B: Condens. Matter Mater. Phys. 2002, 65, No. 161310.

(50) Ohadi, H.; Kammann, E.; Liew, T. C. H.; Lagoudakis, K. G.; Kavokin, A. V.; Lagoudakis, P. G. Spontaneous Symmetry Breaking in a Polariton and Photon Laser. Phys. Rev. Lett. 2012, 109, 016404.

(51) Askitopoulos, A.; Kalinin, K.; Liew, T. C. H.; Cilibrizzi, P.; Hatzopoulos, Z.; Savvidis, P. G.; Berloff, N. G.; Lagoudakis, P. G. Nonresonant optical control of a spinor polariton condensate. Phys. Rev. B: Condens. Matter Mater. Phys. 2016, 93, 205307.

(52) Pickup, L.; Töpfer, J. D.; Sigurdsson, H.; Lagoudakis, P. G. Polariton spin jets through optical control. Phys. Rev. B: Condens. Matter Mater. Phys. 2021, 103, 155302.

(53) Laussy, F. P.; Shelykh, I. A.; Malpuech, G.; Kavokin, A. Effects of Bose-Einstein condensation of exciton polaritons in microcavities on the polarization of emitted light. Phys. Rev. B: Condens. Matter Mater. Phys. 2006, 73, 035315.

(54) Malpuech, G.; Glazov, M. M.; Shelykh, I. A.; Bigenwald, P.; Kavokin, K. V. Electronic control of the polarization of light emitted by polariton lasers. Appl. Phys. Lett. 2006, 88, 111118.

(55) del Valle, E.; Sanvitto, D.; Amo, A.; Laussy, F. P.; André, R.; Tejedor, C.; Viña, L. Dynamics of the Formation and Decay of Coherence in a Polariton Condensate. Phys. Rev. Lett. 2009, 103, 096404.

(56) Kasprzak, J.; André, R.; Dang, L. S.; Shelykh, I. A.; Kavokin, A. V.; Rubo, Y. G.; Kavokin, K. V.; Malpuech, G. Build up and pinning of linear polarization in the Bose condensates of exciton polaritons. Phys. Rev. B: Condens. Matter Mater. Phys. 2007, 75, 045326.

(57) Baumberg, J. J.; Kavokin, A. V.; Christopoulos, S.; Grundy, A. J. D.; Butté, R.; Christmann, G.; Solnyshkov, D. D.; Malpuech, G.; BaldassarriHöger von Högersthal, G.; Feltin, E.; Carlin, J. F.; Grandjean, N. Spontaneous polarization buildup in a room-temperature polariton laser. Phys. Rev. Lett. 2008, 101, 136409.

(58) Martín, M. D.; Ballarini, D.; Amo, A.; Kłopotowsi, Ł.; Viña, L.; Kavokin, A. V.; André, R. Striking dynamics of II-VI microcavity polaritons after linearly polarized excitation. Phys. Status Solidi C 2005, 2, 3880-3883.

(59) Kłopotowski, Ł.; Martín, M. D.; Amo, A.; Viña, L.; Shelykh, I. A.; Glazov, M. M.; Malpuech, G.; Kavokin, A. V.; André, R. Optical anisotropy and pinning of the linear polarization of light in semiconductor microcavities. Solid State Commun. 2006, 139, 511515 . 
(60) Sanvitto, D.; Amo, A.; Viña, L.; André, R.; Solnyshkov, D.; Malpuech, G. Exciton-polariton condensation in a natural twodimensional trap. Phys. Rev. B: Condens. Matter Mater. Phys. 2009, 80, 045301.

(61) Askitopoulos, A.; Nalitov, A. V.; Sedov, E. S.; Pickup, L.; Cherotchenko, E. D.; Hatzopoulos, Z.; Savvidis, P. G.; Kavokin, A. V.; Lagoudakis, P. G. All-optical quantum fluid spin beam splitter. Phys. Rev. B: Condens. Matter Mater. Phys. 2018, 97, 235303.

(62) Gnusov, I.; Sigurdsson, H.; Baryshev, S.; Ermatov, T.; Askitopoulos, A.; Lagoudakis, P. G. Optical orientation, polarization pinning, and depolarization dynamics in optically confined polariton condensates. Phys. Rev. B: Condens. Matter Mater. Phys. 2020, 102, 125419.

(63) Gnusov, I.; Sigurdsson, H. J.; Töpfer, D.; Baryshev, S.; Alyatkin, S.; Lagoudakis, P. G. All-optical linear polarization engineering in single and coupled exciton-polariton Condensates. arXiv: 2101.07602v2 [physics.optics] 2021, No. na.

(64) Ballarini, D.; Amo, A.; Viña, L.; Sanvitto, D.; Skolnick, M. S.; Roberts, J. S. Transition from the strong- to the weak-coupling regime in semiconductor microcavities: Polarization dependence. Appl. Phys. Lett. 2007, 90, 201905.

(65) Kammann, E.; Liew, T. C. H.; Ohadi, H.; Cilibrizzi, P.; Tsotsis, P.; Hatzopoulos, Z.; Savvidis, P. G.; Kavokin, A. V.; Lagoudakis, P. G. Nonlinear optical spin Hall effect and long-range spin transport in polariton lasers. Phys. Rev. Lett. 2012, 109, 036404.

(66) Antón, C.; Morina, S.; Gao, T.; Eldridge, P. S.; Liew, T. C. H.; Martín, M. D.; Hatzopoulos, Z.; Savvidis, P. G.; Shelykh, I. A.; Viña, L. Optical control of spin textures in quasi-one-dimensional polariton condensates. Phys. Rev. B: Condens. Matter Mater. Phys. 2015, 91, 075305 .

(67) Gao, T.; Antón, C.; Liew, T. C. H.; Martín, M. D.; Hatzopoulos, Z.; Viña, L.; Eldridge, P. S.; Savvidis, P. G. Spin selective filtering of polariton condensate flow. Appl. Phys. Lett. 2015, 107, 011106.

(68) Cilibrizzi, P.; Sigurdsson, H.; Liew, T. C. H.; Ohadi, H.; Wilkinson, S.; Askitopoulos, A.; Shelykh, I. A.; Lagoudakis, P. G. Polariton spin whirls. Phys. Rev. B: Condens. Matter Mater. Phys. 2015, 92,155308 .

(69) Cilibrizzi, P.; Sigurdsson, H.; Liew, T. C. H.; Ohadi, H.; Askitopoulos, A.; Brodbeck, S.; Schneider, C.; Shelykh, I. A.; Höfling, S.; Ruostekoski, J.; Lagoudakis, P. G. Half-skyrmion spin textures in polariton microcavities. Phys. Rev. B: Condens. Matter Mater. Phys. 2016, 94, 045315.

(70) Pickup, L.; Kalinin, K.; Askitopoulos, A.; Hatzopoulos, Z.; Savvidis, P. G.; Berloff, N. G.; Lagoudakis, P. G. Optical Bistability under Nonresonant Excitation in Spinor Polariton Condensates. Phys. Rev. Lett. 2018, 120, 225301.

(71) Dasbach, G.; Diederichs, C.; Tignon, J.; Ciuti, C.; Roussignol, Ph.; Delalande, C.; Bayer, M.; Forchel, A. Polarization selective polariton oscillation in quasi $\square$ onedimensionalmicrocavities. Phys. Status Solidi C 2005, 2, 779-782.

(72) Wouters, M.; Carusotto, I. Excitations in a Nonequilibrium Bose-Einstein Condensate of Exciton Polaritons. Phys. Rev. Lett. 2007, 99, 140402.

(73) Winkler, K.; Egorov, O. A.; Savenko, I. G.; Ma, X.; Estrecho, E.; Gao, T.; Müller, S.; Kamp, M.; Liew, T. C. H.; Ostrovskaya, E. A.; Höfling, S.; Schneider, C. Collective state transitions of excitonpolaritons loaded into a periodic potential. Phys. Rev. B: Condens. Matter Mater. Phys. 2016, 93, 121303.

(74) Sedov, E. S.; Rubo, Y. G.; Kavokin, A. V. Zitterbewegung of exciton-polaritons. Phys. Rev. B: Condens. Matter Mater. Phys. 2018, 97, 245312.

(75) Kavokin, A.; Malpuech, G.; Glazov, M. Optical Spin Hall Effect. Phys. Rev. Lett. 2005, 95, 136601.

(76) Shelykh, I. A.; Nalitov, A. V.; Iorsh, I. V. Optical analog of Rashba spin-orbit interaction in asymmetric polariton waveguides. Phys. Rev. B: Condens. Matter Mater. Phys. 2018, 98, 155428.

(77) Weeber, J. C.; Krenn, J. R.; Dereux, A.; Lamprecht, B.; Lacroute, Y.; Goudonnet, J. P. Near-field observation of surface plasmon polariton propagation on thin metal stripes. Phys. Rev. B: Condens. Matter Mater. Phys. 2001, 64, 045411.

(78) Antón, C.; Liew, T. C. H.; Tosi, G.; Martín, M. D.; Gao, T.; Hatzopoulos, Z.; Eldridge, P. S.; Savvidis, P. G.; Viña, L. Energy relaxation of exciton-polariton condensates in quasi-one-dimensional microcavities. Phys. Rev. B: Condens. Matter Mater. Phys. 2013, 88, 035313. 